\title{
Optical versus electron diffraction imaging of Twist-angle in 2D transition metal dichalcogenide bilayers
}

\author{
S. Psilodimitrakopoulos (iD ${ }^{1 凶}$, A. Orekhov ${ }^{2,3}$, L. Mouchliadis ${ }^{1,4}$, D. Jannis ${ }^{2,3}$, G. M. Maragkakis ${ }^{1,5}$, G. Kourmoulakis $^{1,4}$, N. Gauquelin $^{2,3}$, \\ G. Kioseoglou $\mathbb{D i D}^{1,4}$, J. Verbeeck ${ }^{2,3}$ and E. Stratakis $\left(\mathbb{D}^{1,5} \bowtie\right.$
}

Atomically thin two-dimensional (2D) materials can be vertically stacked with van der Waals bonds, which enable interlayer coupling. In the particular case of transition metal dichalcogenide (TMD) bilayers, the relative direction between the two monolayers, coined as twist-angle, modifies the crystal symmetry and creates a superlattice with exciting properties. Here, we demonstrate an all-optical method for pixel-by-pixel mapping of the twist-angle with a resolution of $0.55\left(^{\circ}\right)$, via polarizationresolved second harmonic generation (P-SHG) microscopy and we compare it with four-dimensional scanning transmission electron microscopy (4D STEM). It is found that the twist-angle imaging of WS $_{2}$ bilayers, using the P-SHG technique is in excellent agreement with that obtained using electron diffraction. The main advantages of the optical approach are that the characterization is performed on the same substrate that the device is created on and that it is three orders of magnitude faster than the 4D STEM. We envisage that the optical P-SHG imaging could become the gold standard for the quality examination of TMD superlatticebased devices.

npj 2D Materials and Applications (2021)5:77; https://doi.org/10.1038/s41699-021-00258-5

\section{INTRODUCTION}

Following the discovery of graphene, the appearance of $2 \mathrm{D}$ transition metal dichalcogenides (TMD) significantly broadened the knowledge in the field of $2 \mathrm{D}$ materials, as well as opening potential optoelectronic applications. Besides this, vertical stacks of two TMD monolayers (ML) forming a bilayer demonstrate exciting optoelectronic properties, not present in individual MLs' ${ }^{1}$. In such TMD bilayers, the two constituent MLs may possess different crystal directions creating in their overlapping region a moiré superlattice. The relative direction between the two MLs is called the twist-angle.

Photoluminescence (PL) studies on $\mathrm{MoS}_{2}$ TMD bilayers with different twist-angles reveal an interlayer electronic coupling, which corresponds to an indirect bandgap recombination which varies with twist-angle ${ }^{2}$. Recently, it was also discovered that the moiré periodic potential in twisted $\mathrm{MoS}_{2}$ bilayer can modify the properties of phonons in the respective $\mathrm{ML}$ constituents to generate Raman modes related to moiré phonons ${ }^{3}$. More recent studies revealed the presence of moiré excitons in twisted TMD homo- and hetero-bilayers ${ }^{4-6}$. The moiré pattern in the crystal symmetry of a twisted bilayer can be controlled through the rotation of the adjacent layers. In this context, the twist-angle is regarded as a new degree of freedom, enabling tuning of the physical properties of the TMD superlattices. Consequently by tuning the twist-angle in real-space, the change in the moiré pattern and consequently to the moiré periodic potential, one can control the interlayer coupling in order to obtain the desired superlattice properties. Obviously, the precise characterization of the twist-angle in moiré superlattices is essential for a global understanding and quality control in such 2D material systems, as well as precise tuning of the respective vdW devices' performance.

Although TEM is the most commonly used technique to atomically reconstruct twisted TMD bilayers ${ }^{7}$, it requires tedious sample transfer on TEM grids, which is incompatible with most 2D materials fabrication and characterization techniques. Apart from being technically challenging, this procedure might eventually distort the relative lattice direction and alignment. Twisted TMD bilayers have also been imaged using atomic force microscopy $(\mathrm{AFM})^{8}$, but this requires direct contact with the active area of the bilayer, thus entailing the risk of damaging the sample. While scanning electron microscopy (SEM) techniques do not generally suffer from these limitations, conventional SEM techniques used for crystallographic imaging rely on the detection of backscattered primary electrons, which is not enough to probe mono- or bilayer materials ${ }^{9}$.

As far as the optical techniques are concerned, up to date, the estimation of twist-angle in 2D TMD bilayers has been based either on simple optical microscopy observations, or on the production of SHG signals ${ }^{5,6}$. However, the approaches reported to date do not exhibit high accuracy and cannot image the twistangle over extended bilayer areas. Having a tool that spatially resolves, with high-resolution and minimally invasively, the twistangle in large area vdW heterostructures, would be therefore of great importance in the quality characterization of such structures. In this work, we demonstrate such a technique based on the areal imaging of polarization-resolved SHG (P-SHG) signals from TMD superlattices complemented with theoretical modeling that predicts the SHG signals interference from the respective overlapping areas of twisted-bilayers.

2D TMD materials like $W S_{2}$ MLs belong to the $D_{3 h}$ point symmetry group with broken inversion symmetry along the armchair direction. This lack of inversion symmetry in the TMD ML results in coherent SHG signals, when an intense field is incident on the $2 \mathrm{D}$ material ${ }^{10,11}$. The non-centrosymmetry which creates the SHG signals originates from the honeycomb lattice of the $\mathrm{WS}_{2}$ $2 \mathrm{D}$ crystal, because of the alternating $\mathrm{S}$ and $\mathrm{W}$ atoms (top view in

\footnotetext{
${ }^{1}$ Institute of Electronic Structure and Laser, Foundation for Research and Technology-Hellas, Heraklion Crete, Greece. ${ }^{2}$ Electron Microscopy for Materials Science (EMAT) University of Antwerp, Antwerp, Belgium. ${ }^{3}$ NANOlab Center of Excellence, University of Antwerp, Antwerp, Belgium. ${ }^{4}$ Department of Materials Science and Technology, University of Crete, Heraklion Crete, Greece. ${ }^{5}$ Department of Physics, University of Crete, Heraklion Crete, Greece. ${ }^{凶}$ email: sopsilo@iesl.forth.gr; stratak@iesl.forth.gr
} 
a

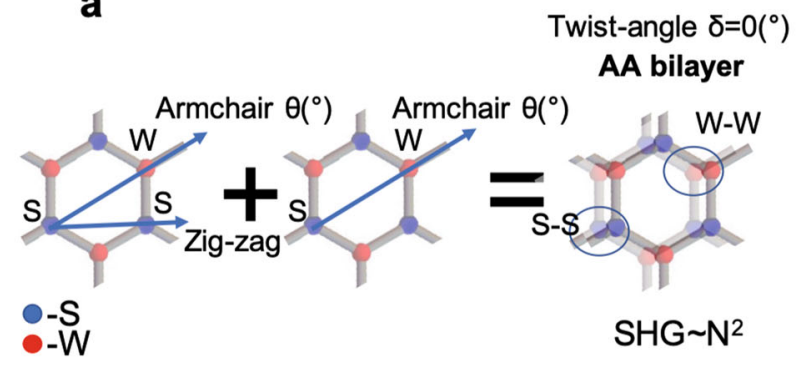

b

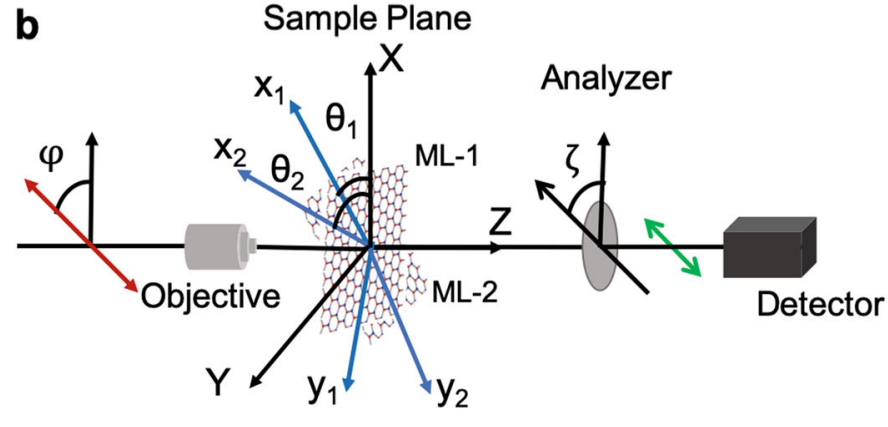

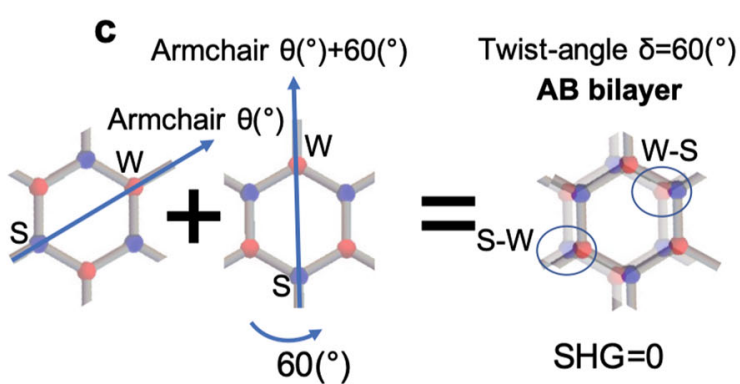

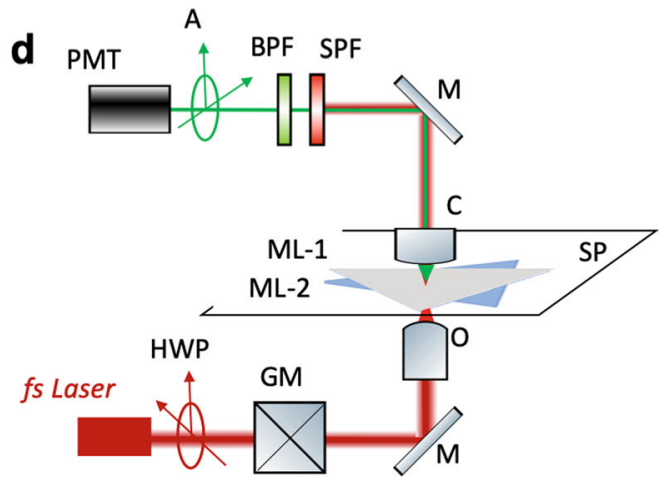

Fig. 1 SHG signals originating from $\mathbf{W S}_{2} / \mathbf{W S}_{2}$ bilayers. a Schematic representation of the top view in the atomic configuration of a $2 \mathrm{D}$ WS bilayer, for the AA stacking sequence. Atoms of W-W or S-S are on top of each other and the SHG signals depend quadratically on the number $\mathrm{N}$ of the MLs $\left(\mathrm{SHG} \sim \mathrm{N}^{2}\right)$. b Coordinate system of the experimental configuration used for P-SHG imaging. $\theta_{1}$ and $\theta_{2}$ denote the armchair directions of ML-1 and ML-2, respectively. $\varphi$ indicates the direction of the excitation linear polarization and $\zeta$ the axis of the analyzer. In our experiments $\zeta$ is fixed to $0\left(^{\circ}\right)$ which greatly simplifies the experimentally retrieval of the P-SHG polar diagrams. c Atomic configuration for the AB stacking sequence. Alternating W-S atoms are on top of each other and the SHG signals cancel (SHG $=0$ ), because centrosymmetry is restored. d Block diagram of the experimental setup used for P-SHG imaging. HWP: half-wave plate, GM: galvanometric mirrors, M: mirror, O: objective, SP: sample plane, C: condenser, SPF: short pass filter, BPF: bandpass filter, A: analyzer, PMT: photomultiplier tube.

bilayer atoms configuration of Fig. 1a). The positions of the alternating $\mathrm{S}$ and $\mathrm{W}$ atoms define the broken symmetry axis of the crystal, which lies in the armchair direction. In contrast, the alternating $\mathrm{S}$ and $\mathrm{S}$ or $\mathrm{W}$ and $\mathrm{W}$ atoms define the zig-zag direction of the crystal.

The generated SHG from a WS $M L$ (crystal class $D_{3 h}$ ) is described by its corresponding susceptibility tensor, $x^{(2)}$. In our approach, we rotate the direction of the linear polarization $\varphi$ of the excitation field and we detect the SHG component parallel to the $X$-axis $\left(\zeta=0\left(^{\circ}\right)\right.$ in Fig. $\left.1 b\right)$. Then, the recorded SHG from a $W_{2}$ $\mathrm{ML}$ is given by (a four-leaved rose-like, polar diagram) ${ }^{12}$

$I_{(\mathrm{ML})}^{2 \omega}=\left[A \cos \left(3 \theta_{1}-2 \varphi\right)\right]^{2}$.

here $A=E_{0}^{2} \varepsilon_{0} X_{x x x}^{(2)}$, with $\varepsilon_{0}$ being the dielectric constant, $E_{0}$ the amplitude of the excitation field and $\theta_{1} \in\left[0\left({ }^{\circ}\right)-60\left(^{\circ}\right)\right]$, defines the armchair direction of the $\mathrm{ML}$ modulo $60\left(^{\circ}\right)$. This means that the armchair directions with $\theta_{1}\left({ }^{\circ}\right)+\mathrm{k} 60\left(^{\circ}\right)$ in Eq. (1) (where $\mathrm{k}$ is an integer) will provide the same P-SHG polar diagram.

When two TMDs, i.e. two $\mathrm{WS}_{2} \mathrm{MLs}$, are stacked to form a bilayer, the SHG signals from each ML interfere and the total SHG intensity from the $\mathrm{WS}_{2} / \mathrm{WS}_{2}$ superlattice is described by

$I_{(\mathrm{BI})}^{2 \omega}=A^{2}\left[\cos \left(3 \theta_{1}-2 \varphi\right)+\cos \left(3 \theta_{2}-2 \varphi\right)\right]^{2}$

Now, one can use the concept of effective armchair direction $\theta_{\text {eff }}$ in the overlapping region of the two $\mathrm{WS}_{2}$ monolayers and express the total SHG intensity produced by the $2 \mathrm{MLs}_{\mathrm{as}^{7}}$

$l_{(\mathrm{BI})}^{2 \omega}=\left[A_{\mathrm{eff}} \cos \left(3 \theta_{\mathrm{eff}}-2 \varphi\right)\right]^{2}$

where

$A_{\text {eff }}=2 A \cos \frac{3}{2} \delta$ where $\delta=\theta_{1}-\theta_{2}$ is the twist-angle, between the MLs and

$\theta_{\text {eff }}=\frac{\theta_{1}+\theta_{2}}{2}$.

As a result, the P-SHG modulation emerging from a bilayer region consisting of two $W S_{2} M L s$, at twist angle $\delta=\theta_{1}-\theta_{2}$, behaves as if it was the P-SHG modulation of a single ML with armchair direction $\theta_{\text {eff. }}$ The $\theta_{\text {eff }}$ can be extracted experimentally from the P-SHG polar obtained from the bilayer region. In the above, the excitation field propagates along the $Z$-axis and the $x_{1}$ armchair direction of WS $\mathrm{WL}_{2}-1$ is at angle $\theta_{1}$ with respect to the $X$ axis (Fig. 1b). While the second $W_{2} M L-2$ has its armchair direction lying in the $x_{2}$ direction, at angle $\theta_{2}$ with respect to the $X$ axis (Fig. 1b).

Note in Eq. (4), that the SHG intensity from the twisted bilayer depends on the twist-angle $\delta$, being maximum for $\delta=0\left(^{\circ}\right)$ and zero for $\delta=60\left(^{\circ}\right)$. When the two $\mathrm{WS}_{2}$ MLs are perfectly aligned, e.g. for $\left(\delta=0\left(^{\circ}\right)\right.$ ), we have the AA stacking sequence (S or W atoms in one layer lie respectively above the $S$ or $W$ atoms of the second layer (S-S, W-W in Fig. 1a). In this case, the total SHG signal from the bilayer is the result of constructive interference, resulting in SHG intensity four times larger than that of the ML (for $N$ number of MLs the produced SHG signal is analogous to $N^{2}$ ). In the case of AB stacking sequence $\left(\delta=60\left(^{\circ}\right)\right)$ the $S$ or W atoms in one layer lie, respectively, above the $W$ or $S$ atoms of the second layer ( $S-W$, $\mathrm{W}-\mathrm{S}$ in Fig. 1c). In this case, centrosymmetry is restored and the SHG signal from the bilayer vanishes $(\mathrm{SHG}=0)$.

The above considerations, refer to the ideal cases of complete constructive or destructive interference in $A A$ and $A B$ bilayer stacking sequences, respectively. However, deviations from these ideal stacking sequences, can occur ${ }^{13}$. A direct consequence of such departures from the stacking sequences $A A$ and $A B$ is the incomplete constructive or destructive interference of the SHG 

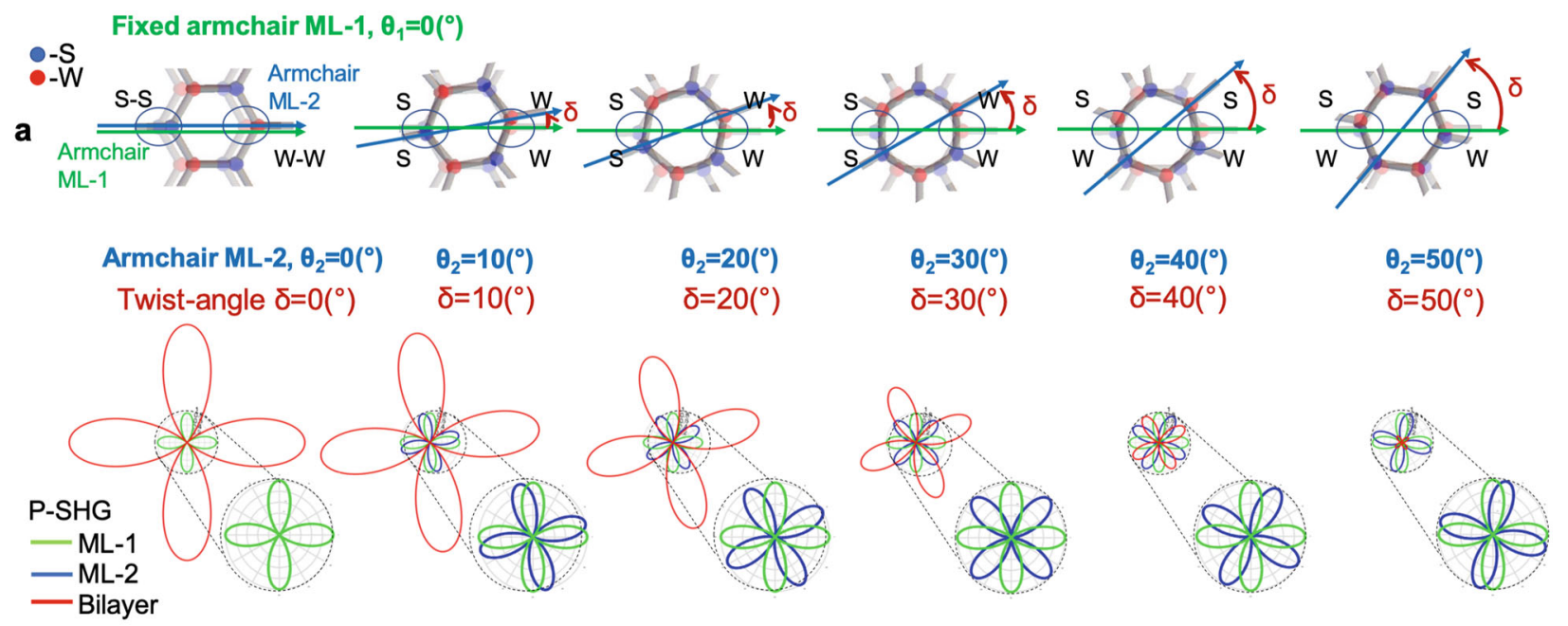

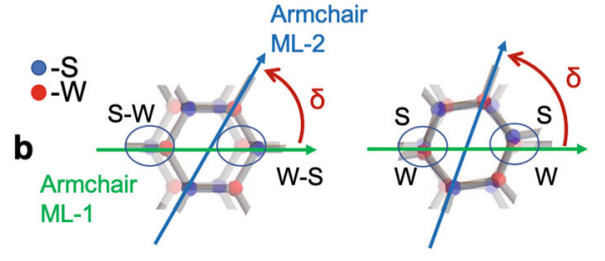

$$
\begin{array}{cc}
\text { Armchair ML-2, } \theta_{2}=60\left(^{\circ}\right) & \theta_{2}=10\left({ }^{\circ}\right)+60\left(^{\circ}\right) \\
\delta=60\left({ }^{\circ}\right) & \delta=70\left(^{\circ}\right)
\end{array}
$$

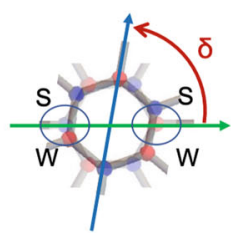

$\theta_{2}=20\left(^{\circ}\right)+60\left({ }^{\circ}\right)$ $\delta=80\left(^{\circ}\right)$
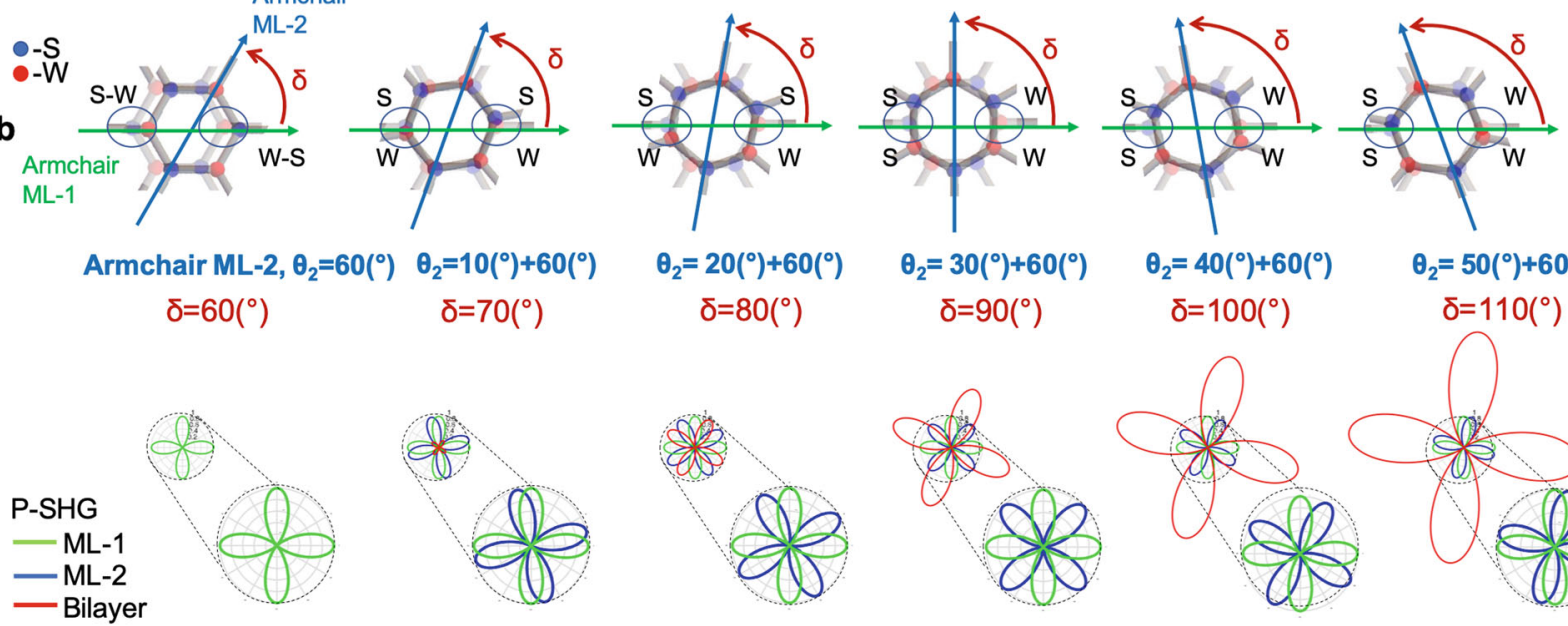

$$
\delta=100\left(^{\circ}\right)
$$
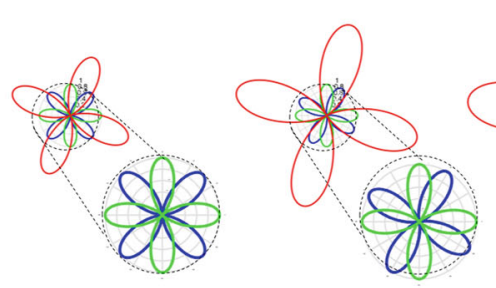

Fig. 2 P-SHG interference signatures in twisted $\mathbf{W S}_{\mathbf{2}} / \mathbf{W S}_{\mathbf{2}}$ bilayers. a Schematic showing the dependence of P-SHG signal on the twist-angle for fixed armchair direction $\mathrm{ML}-1,\left(\theta_{1}=0\left(^{\circ}\right)\right.$ ) and varying armchair direction $\theta_{2}$ of $\mathrm{ML}-2, \theta_{2} \in\left[0\left(^{\circ}\right)-50\left(^{\circ}\right)\right]$, with step $10\left(^{\circ}\right)$. Top view atom configuration hexagonal lattice of the bilayer and simulated polar diagrams of the interference P-SHG originated from the WS ${ }_{2} / \mathrm{WS}_{2}$ bilayer (in red), as well as the simulated P-SHG signal modulation of each individual $\mathrm{WS}_{2}$ ML (green for ML-1 and blue for ML-2). $\mathbf{b}$ P-SHG signal modulation for fixed $\theta_{1}=0\left(^{\circ}\right)$ and varying $\theta_{2} \in\left[60\left(^{\circ}\right)-110\left({ }^{\circ}\right)\right]$, with step $10\left(^{\circ}\right)$. By comparing a with $b$, we notice that the same green and blue P-SHG polar diagrams can provide two different interference P-SHG red polar diagrams. Therefore, we are able to define unequivocally the armchair direction $\theta_{2}$ that contributes to the SHG signal of the bilayer in the range of $0\left(^{\circ}\right) \leq \theta_{2} \leq 120\left(^{\circ}\right)$.

from different MLs. In this case, the P-SHG signal modulation depends on the twist-angle between the MLs as shown in Fig. 2.

The graphical representation of Eq. (1) and the corresponding visualization in a polar diagram (presented in Fig. 2) demonstrates a fourfold symmetry of the P-SHG intensity modulation that rotates for different armchair directions $\theta$. Thus, each armchair direction corresponds to a characteristic fourfold symmetric (fourleaved rose-like) polar diagram. Consequently, we can calculate $\theta$ by fitting Eq. (1) to experimentally retrieved P-SHG signal intensities with analyzer parallel to the $X$-axis and different linear excitation directions $\varphi$. The experimental configuration of a fixed analyzer parallel to the $X$-axis used here $\left(\zeta=0\left(^{\circ}\right)\right.$ in Fig. $\left.1 b\right)$, is much simpler to that of a rotating analyzer parallel to the rotating linear excitation polarization $(\zeta=\varphi$ in Fig. 1b), used previously6.

The green polar diagrams in Fig. 2 correspond to $\theta_{1}=0\left(^{\circ}\right)$ (created using Eq. (1)), while the blue polar diagrams correspond to $0\left(^{\circ}\right) \leq \theta_{2} \leq 110\left(^{\circ}\right)$ with a step of $10\left(^{\circ}\right.$ ) (created again using Eq. (1)). The red polar diagrams correspond to the product of interference P-SHG in the bilayer region (created using Eq. (2)).
By comparing Fig. 2a, with Fig. 2b, we note the effect of the modulo of $60\left(^{\circ}\right)$ in the calculation of the armchair direction of individual MLs. That is, individual MLs with armchair directions $\theta\left(^{\circ}\right)$ $+\mathrm{k} 60\left({ }^{\circ}\right)$, where $k$ is an integer, will provide the same P-SHG polar diagrams. This implies that P-SHG measurements can calculate the armchair direction of an individual $M L$ in the range $0\left(^{\circ}\right)-60\left(^{\circ}\right)$. Nevertheless, in the case of the bilayer, the SHG signals originate from constructive or destructive interference due to the atomic phase matching between the individual MLs (Fig. 2). Thus, the produced P-SHG modulation from the bilayer (calculated using Eq. (2)) dictates a new angle range for the armchair direction $\theta_{2}$ of the second $M L-2$, i.e. $0\left(^{\circ}\right)-120\left(^{\circ}\right)$.

Additionally, as we note in Fig. 2, the SHG signals originating from the bilayers regions with twist-angles of $10\left({ }^{\circ}\right)$ and $110\left({ }^{\circ}\right), 20\left(^{\circ}\right)$ and $100\left({ }^{\circ}\right), 30\left({ }^{\circ}\right)$ and $90\left({ }^{\circ}\right), 40\left(^{\circ}\right)$ and $80\left({ }^{\circ}\right)$, are of equal SHG intensities. Nevertheless, the P-SHG interference polar diagrams from the bilayers' regions (red lines in Fig. 2) for the twist-angles of $10\left(^{\circ}\right)$ and $110\left({ }^{\circ}\right), 20\left(^{\circ}\right)$ and $100\left({ }^{\circ}\right), 30\left(^{\circ}\right)$ and $90\left({ }^{\circ}\right), 40\left(^{\circ}\right)$ and $80\left(^{\circ}\right)$ are different, thus P-SHG is able to identify and discriminate twistangles that produce similar SHG intensities from the bilayer regions. 


\section{RESULTS AND DISCUSSION PSHG measurements}

In order to create the $\mathrm{WS}_{2} / \mathrm{WS}_{2}$ bilayer, two $\mathrm{WS}_{2}$ monolayers were produced by mechanical exfoliation and were stacked with dry stamping on a $\mathrm{Si}_{3} \mathrm{~N}_{4}$ support-grid (see Methods). This allows direct comparison between P-SHG and 4D STEM. The excitation source used for the SHG experiments is an fs oscillator, at $1030 \mathrm{~nm}$ and repetition rate in the order of $\mathrm{MHz}$, which is adequate to excite non-linear signals like SHG (see Fig. $1 \mathrm{~b}$ and Methods for the P-SHG microscope).

In Fig. 3a, a CCD image of two individual $W_{2} S_{2} M L s(M L-1$ and $M L-2)$, forming a $W_{2} / W_{2}$ bilayer on the supporting TEM grid, is shown. One can identify two different types of regions, created by the above stacking, which produce different SHG signals (Fig. 3b, c). Specifically, we identify regions where only one of the two $M L-1$, and $M L-2, W S_{2}$ is present, as well as the region where the two MLs spatially overlap and create the $\mathrm{WS}_{2} / \mathrm{WS}_{2}$ bilayer. We note that, in the absence of an analyzer in the detection path, the SHG intensity from the bilayer region is lower than the SHG from the MLs regions.

In order to calculate the twist-angle of the bilayer, we utilize high-resolution P-SHG measurements with a step of $\varphi=1\left(^{\circ}\right)$. Figure $3 \mathrm{~d}$ presents the respective P-SHG images for fixed analyzer $\left(\zeta=0\left(^{\circ}\right)\right)$ and varying direction of the excitation linear polarization for $\varphi \in\left[0\left(^{\circ}\right)-360\left(^{\circ}\right)\right]$. The corresponding polar diagrams (Fig. 3e, f) obtained from two points of interest (POls 1,2 in Fig. 3c), one for each $M L$, are fitted to Eq. (1) in order to calculate the armchair direction of the MLs. Then, by using the effective armchair $\theta_{\text {eff }}$ obtained from the bilayer ( $\mathrm{POI} 3$ in Fig. $3 \mathrm{c}$ ), we can calculate the twist-angle in the superlattice (using the experimentally retrieved polar of Fig. $3 \mathrm{~g}$ ). We find that $\theta_{1}=44.69\left(^{\circ}\right), R^{2}=0.92$ in the ML-1 region and $\theta_{\text {eff }}=6.88\left({ }^{\circ}\right), R^{2}=0.92$ in the bilayer region.

In previous reports, calculation of twist-angle is performed by measuring the armchair direction of the individual MLs, outside the bilayer region and then deducing their twist angle in their overlapping area, ${ }^{5,6}$. If we follow this strategy we obtain $\delta=\theta_{1}-$ $\theta_{2}=44.69\left(^{\circ}\right)-29.17\left(^{\circ}\right)=15.52\left(^{\circ}\right)$. Nevertheless, as we see in Fig. $2 \mathrm{a}$, a twist-angle of $15.52\left(^{\circ}\right)$ should have resulted to constructive SHG interference, i.e. the SHG signal in the bilayer region should have been more intense than the SHG signals from the MLs. This is not the case in our experimental data, where the SHG signal in the bilayer region is less intense than the individual MLs (Fig. $3 e-g$ ). Consequently, following Fig. $2 \mathrm{~b}$ we should use the modulo $60\left(^{\circ}\right)$ in the calculation of $\theta_{1}$ and obtain, $\theta_{1}=44.69\left(^{\circ}\right)+60\left(^{\circ}\right)=104.69\left(^{\circ}\right)$. As a consequence, we unequivocally determine the twist-angle $\delta=\theta_{1}-\theta_{2}=104.69\left(^{\circ}\right)-29.17\left(^{\circ}\right)=75.52\left(^{\circ}\right)$. We could also use the $\theta_{\text {eff }}$ acquired from the bilayer region and Eq. (5) to retrieve the twist-angle as $\delta=\theta_{1}-\theta_{2}=2\left(\theta_{1}-\theta_{\text {eff }}\right)=2\left(44.69\left(^{\circ}\right)-6.88\left(^{\circ}\right)\right)=$ $75.62\left(^{\circ}\right)$

In Fig. 4b we present pixel-wise mapping of the armchair direction for the region seen in Fig. 4a. We used 360 P-SHG images, each one acquired for $1\left(^{\circ}\right)$ rotating excitation linear polarization for $\varphi \in\left[0\left(^{\circ}\right)-359\left(^{\circ}\right)\right]$ and we have fitted them pixel-bypixel to Eq. (1). We choose to keep only the pixels that exhibit a quality of fitting, $R^{2}>0.8$. As we have explained in ${ }^{12}$ the P-SHG signal fails to fit in the borders among the regions of different armchair orientations in Fig. 4b, c. As a consequence, the pixels located at these borders are missing from the armchair map.

In Fig. 4c we present a magnification of the ROI marked in Fig. 4b. We have also marked two ROls-1,2, one for each ML-1,2, respectively, and $\mathrm{ROI}-3$ in the bilayer region. In Fig. $4 \mathrm{~d}$ we show the image histogram of the armchair directions present in Fig. $4 \mathrm{c}$ and in the ROI seen in Fig. $4 \mathrm{~b}$. In the same Fig. $4 \mathrm{~d}$ we present the mean and the standard deviation $(\sigma)$ values of the armchair directions found in ROls 1-3. We found $\left\langle\theta_{1}\right\rangle=44.64\left(^{\circ}\right)$ with $\sigma=$ $0.04\left(^{\circ}\right),\left\langle\theta_{2}\right\rangle=44.64\left(^{\circ}\right), \sigma=0.06\left(^{\circ}\right)$ and $\left\langle\theta_{\text {eff }}\right\rangle=6.96\left(^{\circ}\right), \sigma=0.55$ $\left({ }^{\circ}\right)$. The iterative fitting procedure resulted in small standard deviation values for ROls 1,3 (Fig. 4c). The very small size of ROls, smaller than the optical resolution of our 1.3NA objective ( $\sim 500 \mathrm{~nm}$ for the $1027 \mathrm{~nm}$ excitation), and the oversampling with many measurements (pixels) inside those small size ROls are responsible for the resulted small standard deviations. The broadening of the angle distributions seen in Fig. $4 \mathrm{~d}$ is attributed to erroneous values originating from the borders of the different regions with different crystal orientations. This is because during the laser raster-scanning the spot of approximately $500 \mathrm{~nm}$ size when passing from those borders, excites SHG from both regions of different crystal orientation. If we follow the strategy of deducing the twist-angle by measuring the armchair direction of the individual MLs we acquire $\left.\delta=44.64\left(^{\circ}\right)-29.22\left(^{\circ}\right)\right)=15.42\left(^{\circ}\right)$. Nevertheless, as we see in Fig. 2 such twist-angle value should have resulted in constructive SHG interference, i.e. the SHG signal in the bilayer region should have been bigger than the SHG signal of the MLs (Fig. 3e-g). This is not the case for our experimental data, therefore we should follow Fig. 2 and use the modulo $60\left(^{\circ}\right)$ in the calculation of $\theta_{1}$ and obtain, $\theta_{1}=44.64\left(^{\circ}\right)+60\left(^{\circ}\right)=104.64\left(^{\circ}\right)$. This enables us to unequivocally determine the twist-angle $\delta=\theta_{1}$ $-\theta_{2}=104.64\left(^{\circ}\right)-29.22\left(^{\circ}\right)=75.42\left(^{\circ}\right)$. We could also use the mean $\theta_{\text {eff }}$ acquired from ROI-3 in the bilayer region and Eq. (5) to retrieve the twist-angle as, $\delta=\theta_{1}-\theta_{2}=2\left(\theta_{1}-\theta_{\text {eff }}\right)=2\left(44.64\left(^{\circ}\right)-6.96\left(^{\circ}\right)\right)$ $=75.36 \pm 0.55\left(^{\circ}\right)$.

\section{D STEM measurements}

4D STEM was employed to measure the crystal directions of the individual monolayers, as well to independently estimate the twist-angle in the $\mathrm{WS}_{2} / \mathrm{WS}_{2}$ bilayer region seen in Fig. 3a. The schematic representation of the application of the 4D STEM method for studying $\mathrm{WS}_{2}$ monolayers is shown in Fig. 5a. The microscope settings are presented in the Methods section. In particular, the bilayer region that has been analyzed by P-SHG is scanned with $256 \times 256$ probe positions with a step size of $25 \mathrm{~nm}$. A virtual dark field image (VDF), calculated by summing the intensity of diffraction spots in each probe position over the selected virtual aperture, is shown in Fig. $5 \mathrm{~b}$. The inner and outer radii of the virtual aperture are chosen to select the second-order diffraction spots of the $\mathrm{WS}_{2}$ structure (Fig. $5 \mathrm{c}$ ). From the VDF intensity distribution, we can identify four distinct regions corresponding to the silicon nitride support-gd (dark contrast) (mean diffraction pattern is shown in Fig. 5c), two monolayers (Fig. $5 \mathrm{~b}, \mathrm{e})$ and an overlapped region with a bilayer (Fig. 5f). At the edges of the monolayers there are regions with increased intensity due to their folding (white arrows in Fig. 5b).

To calculate a direction map of the region, a custom-made peak finding routine was used. Once peak positions are found, two reciprocal vectors can be fitted to describe all diffraction spots defining the relative crystal direction in every probe position. This algorithm works only for diffraction patterns from monolayers. For the bilayer region, four vectors are fitted to each acquired pattern when more than six peaks are detected inside the virtual aperture area. The relative direction map of the region with a bilayer area (white square in Fig. 5b) is shown in Fig. 6a. The tilt angle of every diffraction pattern for two sheets is presented in the histogram Fig. $6 \mathrm{~d}$. The twist-angle between the two monolayers was calculated with subpixel accuracy by fitting a Gaussian function for each peak on the histogram and was found to be $15.5 \pm 0.3\left(^{\circ}\right)$. These results are in good agreement with the experiments on the same area using the all-optical P-SHG imaging microscopy technique, presented above.

However, for the direction determination only the positions of the diffraction spots were used without taking into account their intensity. By looking at the diffraction spot symmetry, one might think that $W_{2}$ crystal have six-fold symmetry along the [001] direction, however, due to dynamic electron scattering in combination with the non-centrosymmetry of the crystal, Friedel's 
a

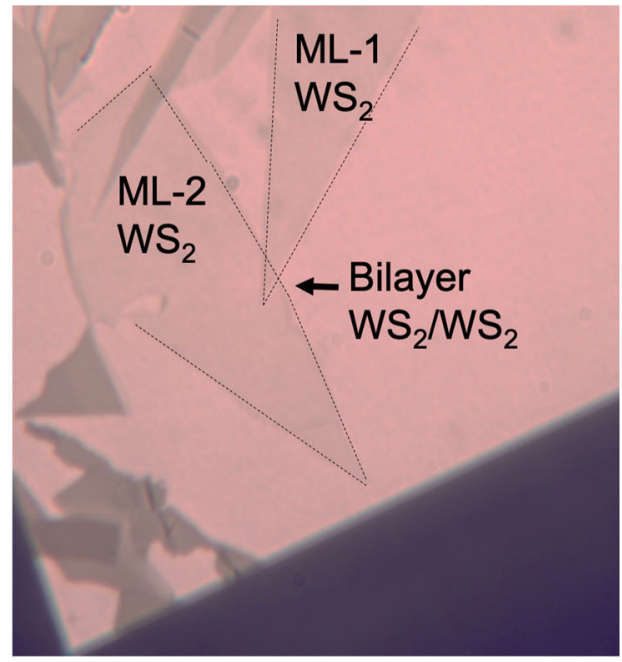

C

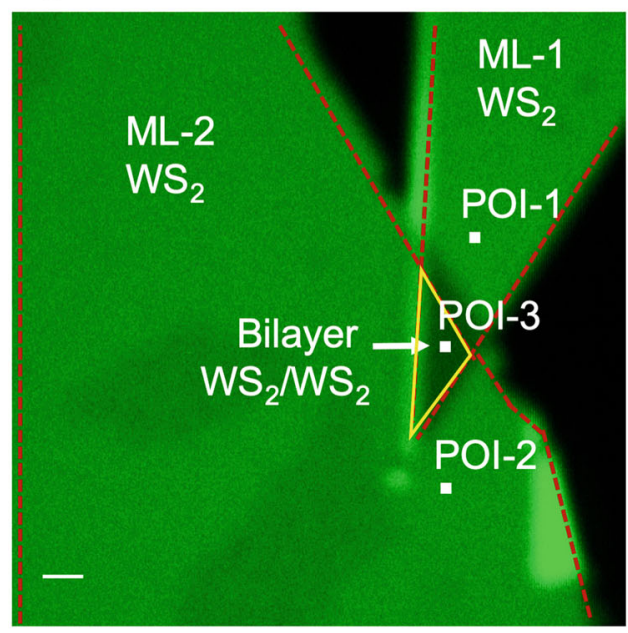

b

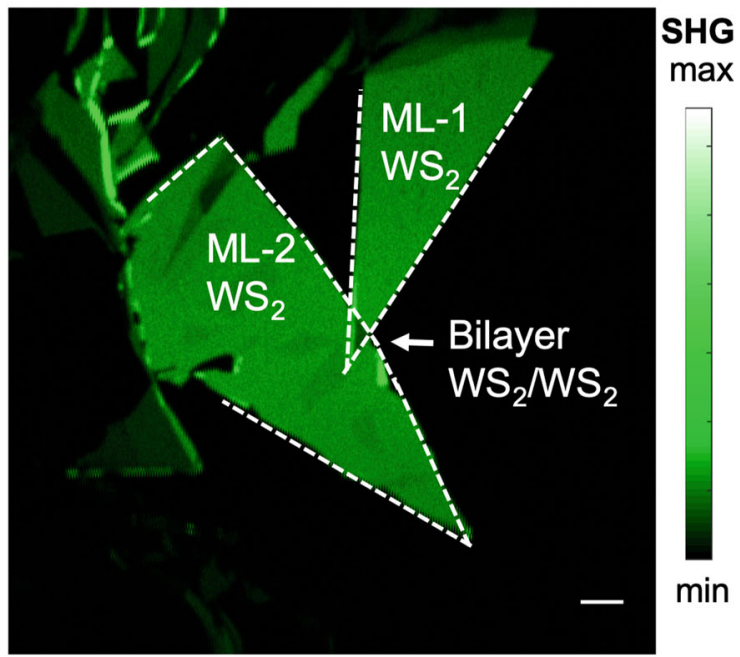

d
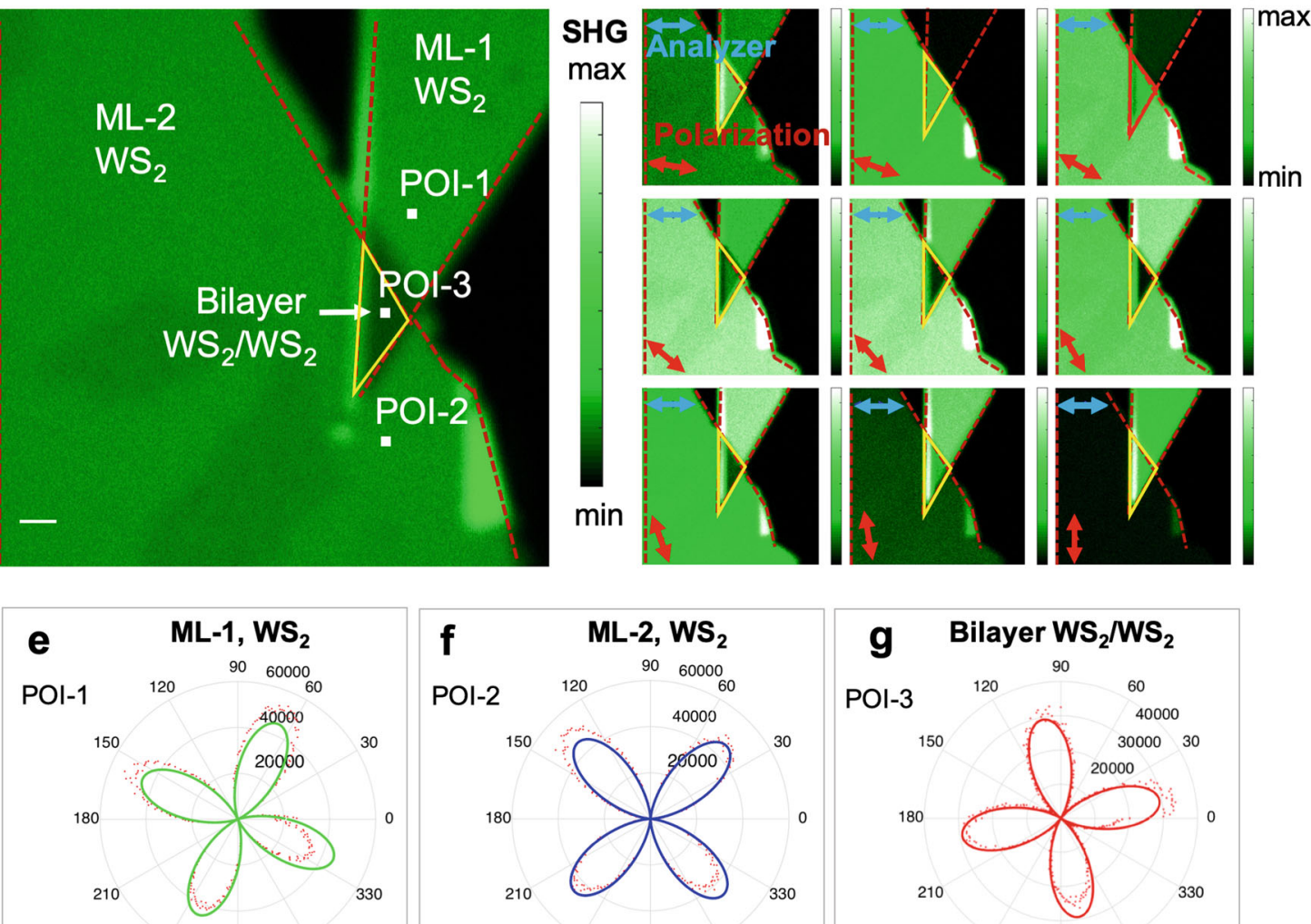

$$
\begin{aligned}
& \mathrm{R}^{2}=0.92^{240} \\
& \theta_{1}=44.69\left(^{\circ}\right)
\end{aligned}
$$

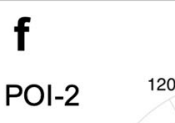

150

180

210

ML-2, WS $_{2}$

$90 \quad 60000$
60

60

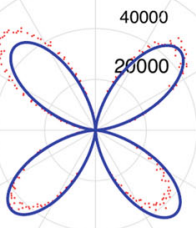

\begin{abstract}
300
$\theta_{2}=29.17\left(^{\circ}\right)$
\end{abstract}

g
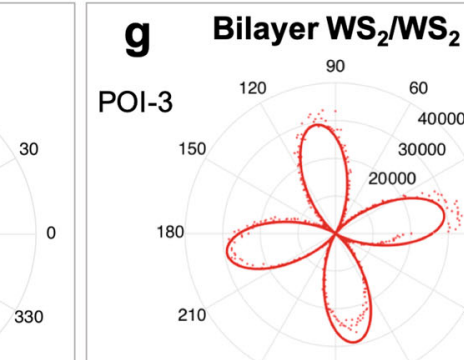

Fig. 3 P-SHG microscopy of $\mathbf{W S}_{\mathbf{2}} / \mathbf{W S}_{\mathbf{2}}$ bilayer on a TEM grid. a Wide field microscopy using the CCD camera of the microscope. We note two individual MLs of WS $(M L-1$ and $M L-2)$, overlapping in a bilayer $\mathrm{WS}_{2} / \mathrm{WS}_{2}$ region. $\mathbf{b}$ SHG imaging of the same region seen in a., without the use of the analyzer in the detection path (see Fig. 1b, d). This ensures that all of the produced SHG signals will be acquired in the same image. Scale bar indicates $5 \mu \mathrm{m}$. c A zoom of the SHG image seen in (b). Three pixels-of-interest (POI) are chosen. One from the WS 2 ML-1, one from the $\mathrm{WS}_{2} \mathrm{ML}-2$ and one from the $\mathrm{WS}_{2} / \mathrm{WS}_{2}$ bilayer region. Scale bar shows $1 \mu \mathrm{m}$. d P-SHG images of the region seen in c. for fixed analyzer $(\zeta=0$ $\left({ }^{\circ}\right)$ ), indicated by a blue double arrow and rotating linear excitation polarization $\left(\varphi \in\left[10\left(^{\circ}\right)-90\left(^{\circ}\right)\right]\right.$, with step $10\left(^{\circ}\right)$ ), indicated by a red double arrow. e, $\mathbf{f}$ Experimentally retrieved P-SHG polar diagrams for the POls 1,2 , respectively. The polarization rotates with $\varphi \in\left[0\left(^{\circ}\right)-360\left({ }^{\circ}\right)\right]$, and step $1\left(^{\circ}\right)$ and the analyzer is fixed at $\zeta=0\left(^{\circ}\right)$. The solid lines (green for ML-1 and blue for ML-2) are the fittings of Eq. (1) to the P-SHG data. This resulted to armchair directions $\theta_{1}=44.69\left(^{\circ}\right), R^{2}=0.92$ and $\theta_{2}=29.17\left(^{\circ}\right), R^{2}=0.96$ for $\mathrm{ML}-1$ and $\mathrm{ML}-2$, respectively, where $R^{2}$ denotes the quality of the fitting. $\mathbf{g}$ Experimentally retrieved P-SHG data for POI 3 in the $\mathrm{WS}_{2} / \mathrm{WS}_{2}$ bilayer region using the same rotating polarization $\varphi \in[0$ $\left({ }^{\circ}\right)-360\left(^{\circ}\right)$, with step $1\left(^{\circ}\right)$ and fixed analyzer at $\zeta=0\left(^{\circ}\right)$ as in $(\mathbf{e}, \mathbf{f})$. The solid red line is the fitting of Eq. $(3)$ to the P-SHG data and $\theta_{\text {eff }}=6.88\left({ }^{\circ}\right)$, $R^{2}=0.92$ is the result of the fitting. 


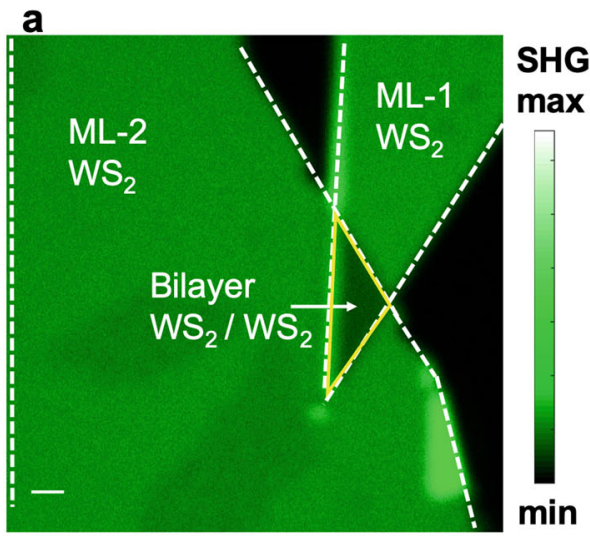

b
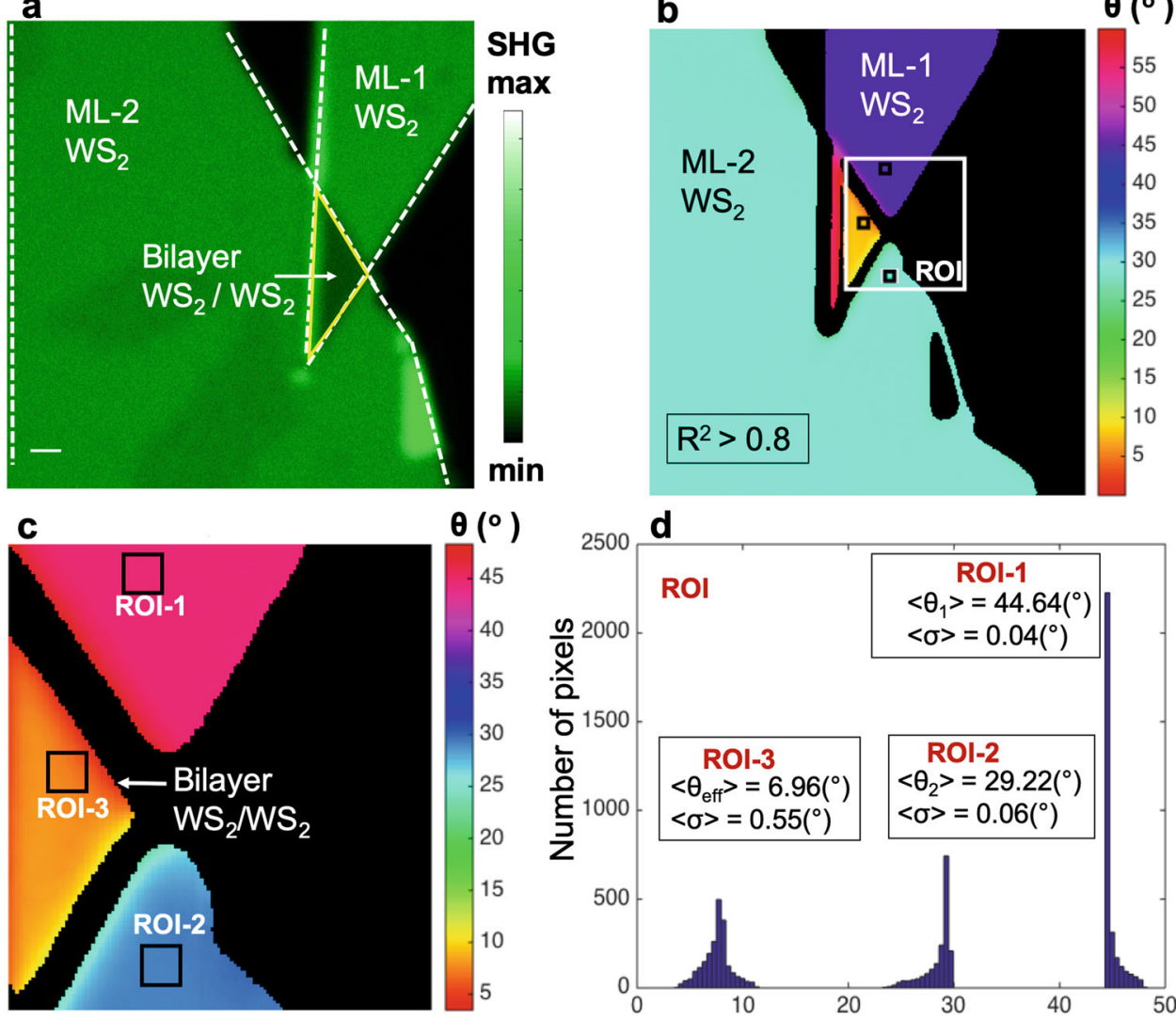

d

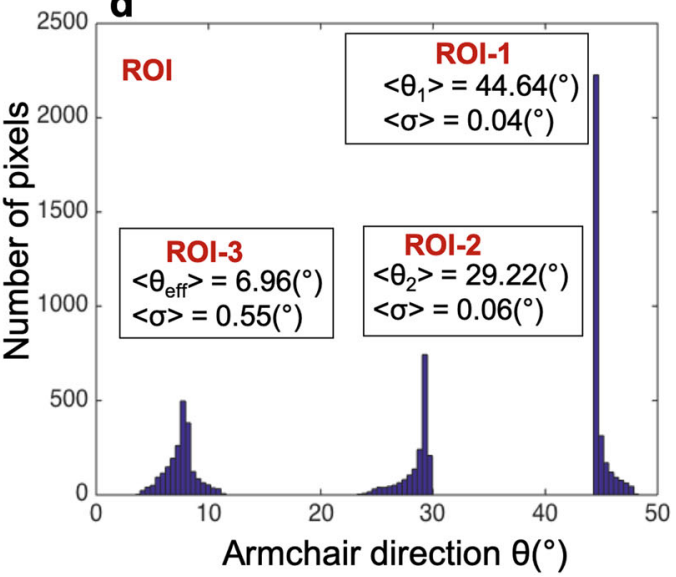

Fig. 4 Optical imaging of twist-angle in $\mathbf{W} \mathbf{S}_{2} / \mathbf{W S}_{2}$ bilayer. a SHG image without the use of analyzer, showing the two ML-WS 2 regions and the bilayer $\mathrm{WS}_{2} / \mathrm{WS}_{2}$ region. We note that the bilayer region has less SHG signal than the MLs regions. Scale bar indicates $1 \mu \mathrm{m}$. $\mathbf{b}$ Mapping of the armchair direction, obtained via pixel-by-pixel fitting of 360 P-SHG images, one for every $\varphi \in\left[0\left(^{\circ}\right)-359\left(^{\circ}\right)\right]$, with step $1\left(^{\circ}\right)$ and fixed analyzer $\left(\zeta=0\left(^{\circ}\right)\right.$ ) to Eq. (1). Only pixels that presented $R^{2}>0,8$ are kept. c Pixel-by-pixel mapping of the armchair direction for the ROI seen in (b). Three ROls are defined. ROIs-1,2 for the WS $\mathrm{WLS}_{2}$ and ROI-3 inside the bilayer $\mathrm{WS}_{2} / \mathrm{WS}_{2}$ region. $\mathbf{d}$ Image histogram showing the distribution of armchair directions seen in (c) and in the ROI seen in (b). The mean armchair directions and their standard deviation $(\sigma)$ of the armchair values found in ROls1-3 are presented.

law is violated resulting in a symmetry reduction to three-fold symmetry and a nonequivalent angular range from $0\left(^{\circ}\right)$ to $120\left(^{\circ}\right)$ instead of $0\left(^{\circ}\right)$ to $60\left(^{\circ}\right)$. Figure $6 \mathrm{c}$, d shows diffraction patterns of the first and second monolayer together with their azimuthal intensity profiles of the first order spots (Fig. 6e) clearly showing 3 fold symmetry. This allows us to identify whether the twist angle is $15.5\left(^{\circ}\right)$ or $75.5\left(^{\circ}\right)\left(15.5\left(^{\circ}\right)+60\left(^{\circ}\right)\right)$ by comparing to simulated diffraction patterns shown in Fig. $6 f, g$. The simulation is performed using the Multem software ${ }^{14,15}$ where the experimental conditions such as convergence angle and electron beam energy are chosen to closely resemble the experimental setup (see Methods section). A noticable difference between the simulated and experimental patterns (Fig. $6 \mathrm{~h}$ ) is caused by the presence of the $\mathrm{Si}_{3} \mathrm{~N}_{4}$-support film (experimental), which gives rise to an isotropic background signal. As can be seen in the simulated patterns the intensity of the first order spots changes azimuthally from two low, two high for the twist angle $15.5\left(^{\circ}\right)$, while it has alternating low-high character for the $75.5\left(^{\circ}\right)$ angle. The annular intensity profile of the first order spots of the experimental pattern (Fig. 6i), shows a clear signature of a $75.5 \pm 0.3\left(^{\circ}\right)$ twist angle and rules out the $15.5 \pm 0.3\left(^{\circ}\right)$ hypothesis. This result is in excellent agreement with the P-SHG data presented above.

In terms of precision, the STEM data demonstrates $0.3\left(^{\circ}\right)$ rotational precision compared to $0.55\left(^{\circ}\right)$ for the P-SHG optical result with a significantly higher spatial resolution but with the downside of a technique which is far less attractive to employ in an inline production environment as compared to the all optical setup proposed in this paper.

Although P-SHG is fast, minimally invasive and can cover large areas, it cannot offer the atomic resolution of the STEM technique. In addition, SHG requires breaking of the symmetry and therefore vanishes in intrinsically centrosymmetric systems (such as graphene) or in systems where centrosymmetry is restored e.g. due to the number of layers (i.e., 2H-stacked TMDs with even number of layers) or e.g to homo-bilayers with twist-angle of $60\left(^{\circ}\right)$. For the case of encapsulated TMDs (e.g. between hBN layers) the collected SHG signal from the overlapping region may include additional contributions (as in the case of hBN which is a polar material), and an additional measurement in an uncovered region is needed to unequivocally determine the relative crystal orientation of the individual layers comprising the bilayer.

Considering that the electronic and optical properties of 2D TMD bilayers can be tuned by changing their twist-angle, a robust and minimally invasive tool that can provide spatially resolved determination of the twist-angle, would be of great importance in research, production and large-scale characterization of 2D TMD bilayers. Here, we have used all-optical, laser raster-scanning, P-SHG imaging microscopy to precisely map the twist-angle in large areas of overlapping $\mathrm{WS}_{2} / \mathrm{WS}_{2}$ stacked monolayers and we benchmarked the results against 4D STEM electron microscopy. It is found that the twist-angle of $\mathrm{WS}_{2} / \mathrm{WS}_{2}$ bilayer obtained using P-SHG mapping is in excellent agreement, either in value and in 

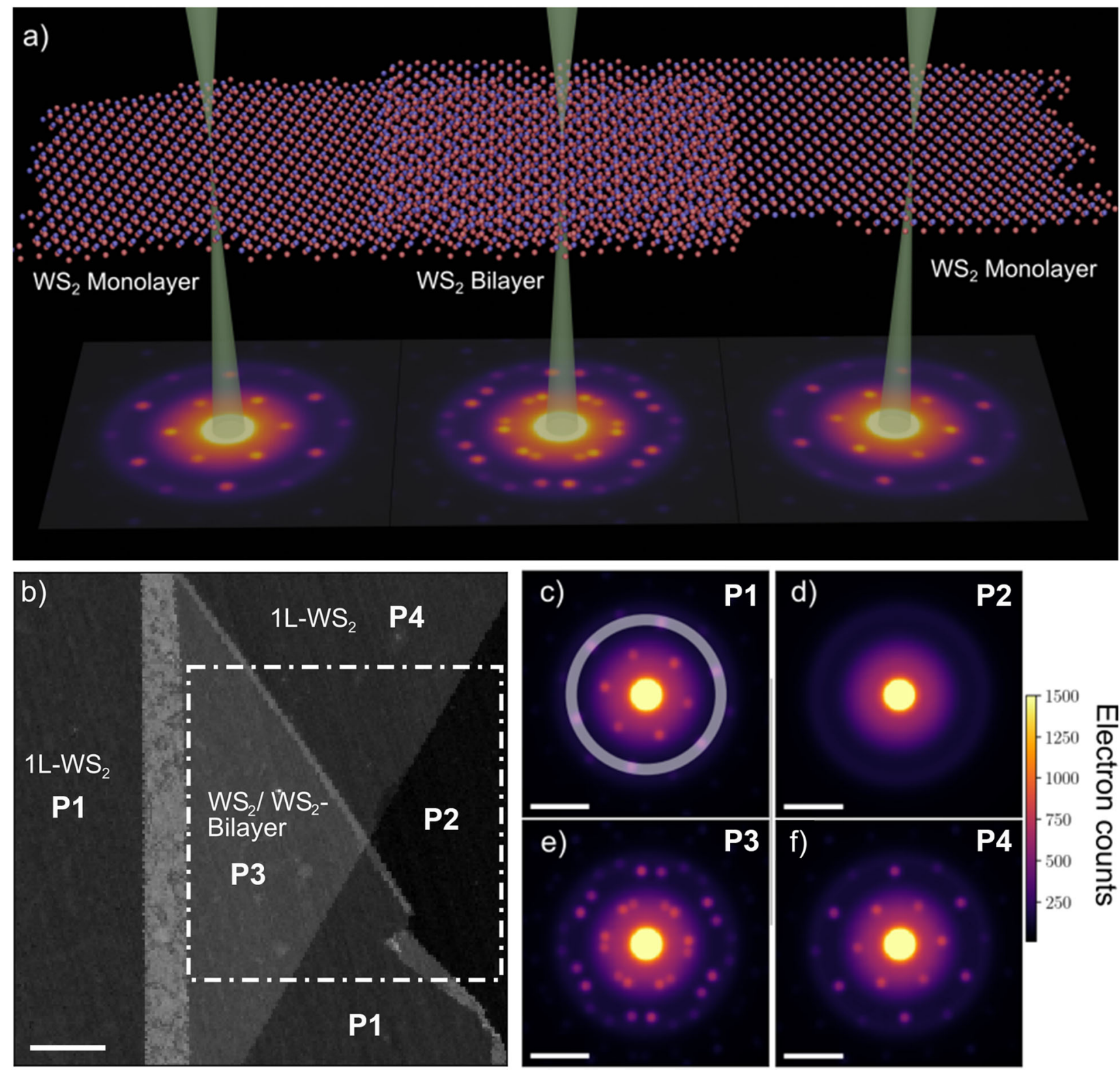

Fig. 5 4D STEM measurements. a Schematic representation of the application of the 4D STEM method for studying WS $\mathrm{S}_{2}$ monolayers. b Virtual dark field image where the intensity in each probe position is summed over selected virtual aperture indicated in (c). Scale bar $1 \mu$ m. c-f The mean diffraction from the four regions (P1-4). c The first $\mathrm{WS}_{2}$ monolayer (P1). d Silicon nitride supporting film (P2). e The bilayer region P3. f The second monolayer P4. Scale bars, $0.5 \AA^{-1}$.

precision, with that obtained using 4D STEM. It is additionally revealed that, given that the produced SHG signal from a bilayer is the vectorial addition of the SHG signals of the individual monolayers, the intensity modulation of the P-SHG signal can be used to deduce unequivocally the armchair direction. This is also in excellent agreement with 4D STEM microscopy analysis.

While STEM provides significantly higher spatial resolution, the P-SHG compensates for this with a wide range of advantages including: no need for vacuum, wide field of view, rapid data acquisition, significantly lower cost and instrument size, and most importantly its capability to work on TMD bilayers deposited on substrates without the need to transfer the films to a TEM grid. Our setup provides an accurate and robust all-optical twist-angle mapping of 2D TMD bilayers. Importantly, the technique is non-destructive, paving the way or directly correlating local twistangle values with electronic properties, which is crucial for the development and scaling up of vdW bilayer devices with precisely controlled functionality.

\section{METHODS}

\section{$\mathrm{WS}_{\mathbf{2}} / \mathrm{WS}_{\mathbf{2}}$ bilayer fabrication on a TEM grid}

Polydimethylsiloxane films (PDMS) were fabricated from 10:1 mixing ratio (SYLGARD 182 Silicone Elastomer Kit) with heat cure at $80^{\circ} \mathrm{C}$ for two hours. High quality WS $\mathrm{S}_{2}$ bulk crystals (HQ Graphene) were mechanically exfoliated directly on the aforementioned PDMS films. The films were placed on typical microscope glass slides using standard protocol ${ }^{16,17}$. Monolayers of these crystals were realized under an optical microscope. In order to produce the bilayer, at first, a glass slide with a WS 2 monolayer was mounted on a XYZ micromechanical stage under a coaxially illuminated microscope and transferred on a silicon nitride $\left(\mathrm{Si}_{3} \mathrm{~N}_{4}\right)$ support-grid using viscoelastic stamping ${ }^{18}$. Finally, another $\mathrm{WS}_{2}$ monolayer was stamped on the previous one in a partial overlapping manner allowing for comparative P-SHG and 4D STEM imaging. 

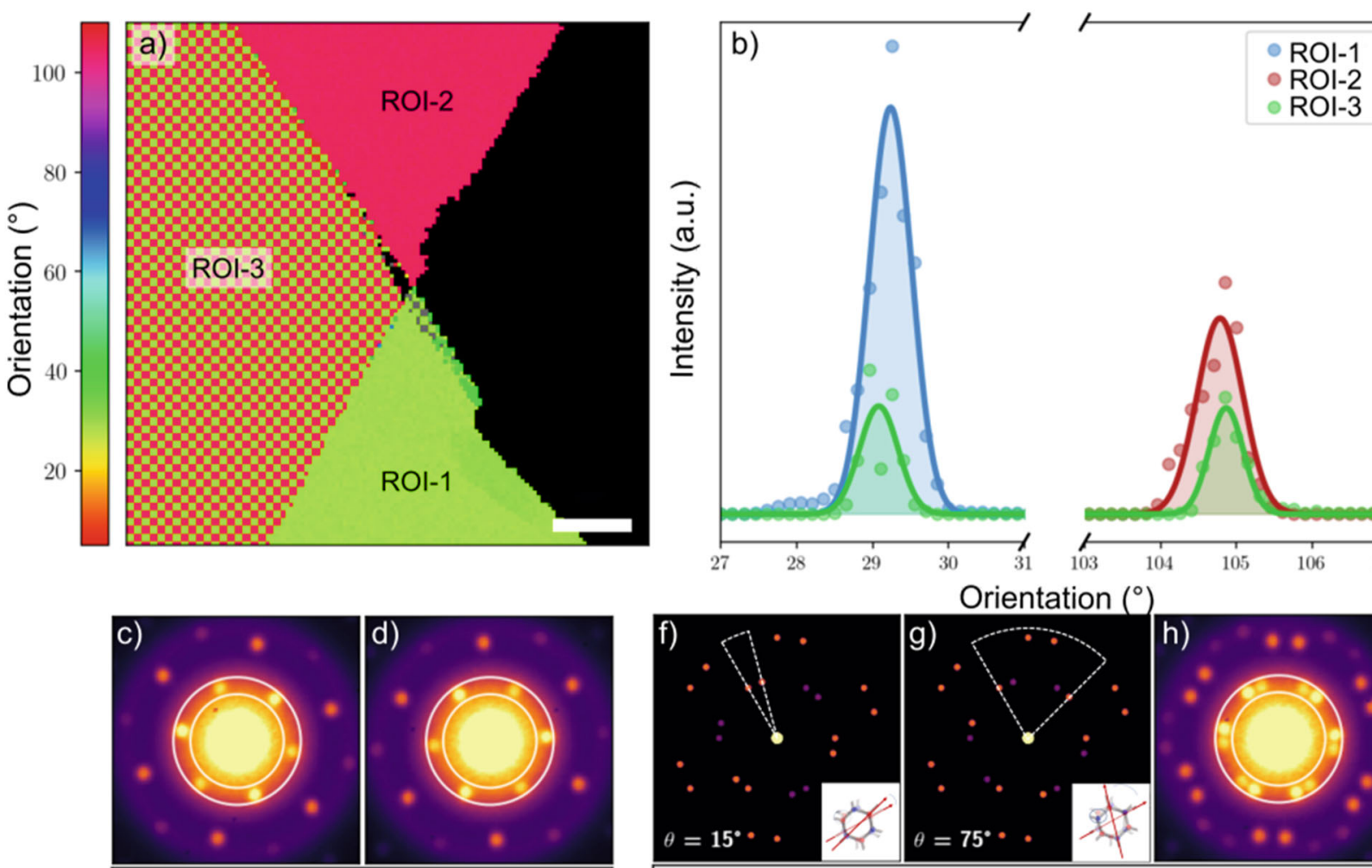

Orientation ( $\left.{ }^{\circ}\right)$
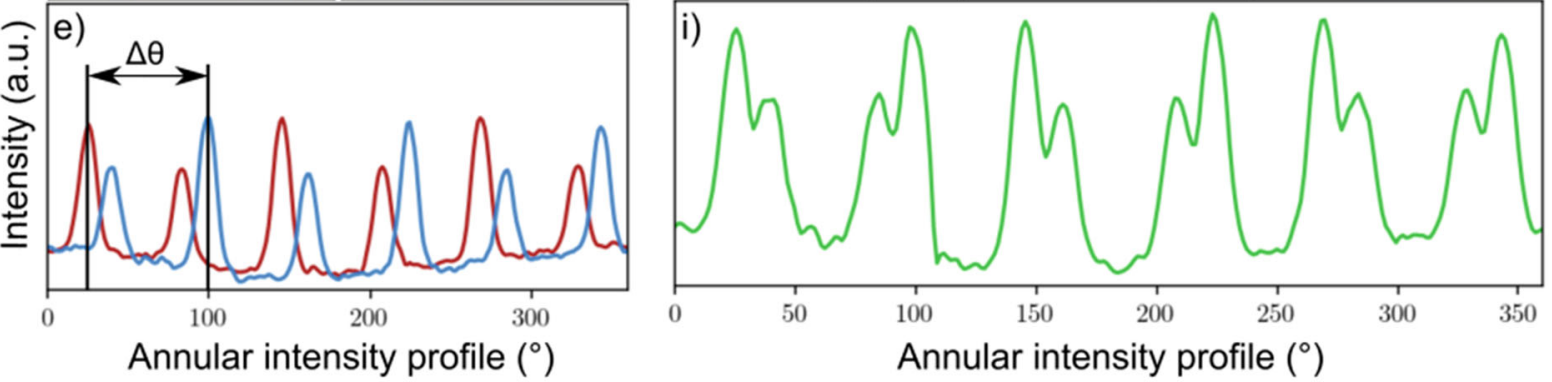

Fig. 6 Twist-angle calculation. a The relative color-coded direction map of a bilayer region selected in Fig. $6 \mathrm{~b}$. $\mathbf{b}$ Histogram of the relative direction of the monolayers and the bilayer indicating that the two layers are stacked upon each other since its direction in the bilayer correspond to both monolayers. c, d The diffraction pattern averaged over 400 probe position for the first and second monolayer. e The annular intensity profile of the first order spots where a modulation of intensity is observed. $\mathbf{f}, \mathbf{g}$ The simulated diffraction patterns of the WS ${ }_{2}$ bilayer when the twist-angle is $15.5\left(^{\circ}\right)$ and $75.5\left(^{\circ}\right)\left(60\left(^{\circ}\right)+15.5\left(^{\circ}\right)\right)$. h Experimental diffraction of the bilayer region, averaged over 400 probe positions. $\mathbf{i}$ The annular intensity profile of the first order spots of experimental diffraction where the intensity modulation of the two layers is reversed indicating the twist angle of $75.5\left(^{\circ}\right)$ instead of $15.5\left(^{\circ}\right)$.

\section{Custom-built P-SHG microscope}

SHG imaging was performed in the forward-detection geometry using a custom-built laser raster-scanning microscope ${ }^{7,11,12}$. As shown schematically in Fig. 1d, a diode-pumped Yb:KGW fs oscillator $(1027 \mathrm{~nm}, 90 \mathrm{fs}$, $76 \mathrm{MHz}$, Pharos-SP, Light Conversion, Lithuania), was inserted into a modified, inverted microscope (Zeiss Axio Observer Z1, Germany), after passing through a pair of silver-coated galvanometric mirrors $(6215 \mathrm{H}$, Cambridge Technology, UK). A motorized rotation stage (M-060.DG, Physik Instrumente, Karlsruhe, Germany), holding a zero order $\lambda / 2$ wave plate (QWPO-1030-10-2, CVI-Laser, USA) was used to rotate the direction of the excitation linear polarization. Then, the beam was reflected on a silver mirror at $45\left(^{\circ}\right)$ (PFR10-P01, ThorLabs, Germany), placed at the turret box of the microscope, just before the objective (Plan Apochromat $40 \times 1.3 \mathrm{NA}$, Zeiss, Germany). The SHG signals were collected from a high-numerical aperture (1.4NA) condenser lens (Zeiss) and guided into a photomultiplier tube (PMT) detector (H9305-04, Hamamatsu, Japan) using another silver mirror (CM1-P01, ThorLabs, Germany) at $45\left(^{\circ}\right)$. The polarization extinction ratio was 28:1. In front of the PMT, a home-built mount was holding a bandpass filter (FF01-514/3-25, Semrock, USA) and a short pass filter (FF01680/SP-25, Semrock, USA), appropriate for SHG imaging. After the filters, a film polarizer (LPVIS100- MP, ThorLabs) was inserted just in front of the PMT to measure the anisotropy of the SHG signals due to the rotation of the excitation linear polarization. Coordination of PMT recordings with the galvo-mirrors movements and with all the motors, as well as the image formation, was performed using LabView (National Instruments, USA). All of our SHG images were of $500 \times 500$ pixels and approximately $1.1 \mathrm{~s}$ was required for each image to be recorded. This resulted in pixel dwell time of approximately $4.4 \mu \mathrm{s}$.

\section{D STEM measurements}

In order to determine local information on the single-layer direction and the bilayer twist angle of 2D materials, a scanning transmission electron microscope ThermoFisher Scientific (FEl) Titan X-Ant-EM was used. The electron microscope was operated in microprobe STEM mode at $300 \mathrm{kV}$ at a convergence semi-angle $a$ of 1 mrad resulting in a probe size of $1.2 \mathrm{~nm}$ in diameter. Local information was obtained by scanning the electron probe over the sample and acquiring an electron diffraction pattern at every probe position ${ }^{19-24}$ on a Medipix3 hybrid pixel direct electron detector (Quantum Detectors Merlin) ${ }^{25-27}$ with a camera length of $115 \mathrm{~mm}$ and exposure time of $5 \mathrm{~ms}$. In comparison to the SHG pixel dwell time of $4.4 \mu \mathrm{s}$, the $5 \mathrm{~ms}$ are three orders of magnitude slower. This detector offers a high frame rate and high efficiency enabling the detection of individual electrons without dark or read-out noise and offering 24 bit dynamic 
range. The diffraction data were processed by custom-made scripts based on the open-source python library Pixstem ${ }^{28}$.

\section{DATA AVAILABILITY}

The data that support this study are available from the corresponding authors upon request.

Received: 12 April 2021; Accepted: 24 July 2021;

Published online: 09 September 2021

\section{REFERENCES}

1. He, J., Hummer, K. \& Franchini, C. Stacking effects on the electronic and optical properties of bilayer transition metal dichalcogenides $\mathrm{MoS}_{2}, \mathrm{MoSe}_{2}, \mathrm{WS}_{2}$, and WSe 2 . Phys. Rev. B 89, 075409 (2014).

2. Liu, K. H. et al. Evolution of interlayer coupling in twisted molybdenum disulfide bilayers. Nat. Commun. 5, 4966 (2014).

3. Lin, M. L. et al. Moiré phonons in twisted bilayer $\mathrm{MoS}_{2}$. ACS Nano 12, 8770 (2018).

4. Jin, $C$. et al. Observation of moire excitons in $\mathrm{WSe}_{2} / \mathrm{WS}_{2}$ heterostructure superlattices. Nature 567, 76-80 (2019).

5. Seyler, K. L. et al. Signatures of moire-trapped valley excitons in $\mathrm{MoSe}_{2} / \mathrm{WSe}_{2}$ heterobilayers. Nature 567, 66-70 (2019).

6. Alexeev, E. M. et al. Resonantly hybridized excitons in moire superlattices in van der Waals heterostructures. Nature 567, 81-86 (2019).

7. Weston, A. et al. Atomic reconstruction in twisted bilayers of transition metal dichalcogenides. Nat. Nanotechnol. 15, 592-597 (2020).

8. Rosenberger, M. R. et al. Twist angle dependent atomic reconstruction and Moiré patterns in transition metal dichalcogenide heterostructures. ACS Nano 14, 4550-4558 (2020).

9. Schwartz, A. J., Kumar, M., Adams, B. L. \& Field, D. P. Electron Backscatter Diffraction in Materials Science (Springer, 2000).

10. Psilodimitrakopoulos, $\mathrm{S}$. et al. Twist Angle mapping in layered $\mathrm{WS}_{2}$ by polarization-resolved second harmonic generation. Sci. Rep. 9, 14285 (2019).

11. Maragkakis, G. M. et al. Imaging the crystal direction of $2 \mathrm{D}$ transition metal dichalcogenides using polarization-resolved second-harmonic generation. OptoElectron Adv. 2, 190026 (2019).

12. Psilodimitrakopoulos, S. et al. Ultrahigh-resolution non-linear optical imaging of the armchair direction in 2D transition metal dichalcogenides. Light Sci. Appl. 7, 18005 (2018).

13. Hsu, W.-T. et al. Second harmonic generation from artificially stacked transition metal dichalcogenide twisted bilayers. ACS Nano 8, 2951-2958 (2014).

14. Lobato, I. \& Van Dyck, D. MULTEM: A new multislice program to perform accurate and fast electron diffraction and imaging simulations using Graphics Processing Units with CUDA. Ultramicroscopy 156, 9-17 (2015).

15. Lobato, I., Van Aert, S. \& Verbeeck, J. Progress and new advances in simulating electron microscopy datasets using MULTEM. Ultramicroscopy 168, 17-27 (2016).

16. Frisenda, R. et al. Recent progress in the assembly of nanodevices and van der Waals heterostructures by deterministic placement of 2D materials. Chem. Soc. Rev. 47, 53-68 (2018).

17. Jayasena, B. \& Melkote, S. N. An Investigation of PDMS stamp assisted mechanical exfoliation of large area graphene. Procedia Manuf. 1, 840-853 (2015).

18. Castellanos-Gomez, A. et al. Deterministic transfer of two-dimensional materials by all-dry viscoelastic stamping. 2D Mater. 1, 011002 (2014).

19. Ophus, C. Four-dimensional scanning transmission electron microscopy (4DSTEM): from scanning nanodiffraction to Ptychography and beyond. Microsc. Microanal. 25, 563-582 (2019).

20. Midgley, P. \& Johnstone, D. Scanning electron diffraction-crystal mapping at the nanoscale. Microsc. Microanal. 24, 182-183 (2018).

21. Yang, H. et al. Simultaneous atomic-resolution electron ptychography and Z-contrast imaging of light and heavy elements in complex nanostructures. Nat. Commun. 7, 12532 (2016).

22. Nederlof, I., van Genderen, E., Li, Y.-W. \& Abrahams, J. P. A Medipix quantum area detector allows rotation electron diffraction data collection from submicrometre three-dimensional protein crystals. Acta Crystallogr. D: Biol. Crystallogr. 69, 1223-1230 (2013).

23. Müller-Caspary, K. et al. Atomic-scale quantification of charge densities in twodimensional materials. Phys. Rev. B 98, 121408 (2018).

24. Nord, M. et al. Strain anisotropy and magnetic domains in embedded nanomagnets. Small 15, 1904738 (2019).

25. Mir, J. A. et al. Characterisation of the Medipix 3 detector for 60 and $80 \mathrm{keV}$ electrons. Ultramicroscopy 182, 44-53 (2017).
26. Plackett, R. et al. Merlin: a fast versatile readout system for Medipix3. J. Instrum. 8 C01038-C01038 (2013).

27. Ballabriga, R., Campbell, M., Heijne, E. H. M., Llopart, X. \& Tlustos, L. The Medipix3 prototype, a pixel readout chip working in single photon counting mode with improved spectrometric performance. IEEE Trans. Nucl. Sci. 54, 1824-1829 (2007)

28. Nord, M. et al. Fast pixelated detectors in scanning transmission electron microscopy. I: Data acquisition, live processing and storage. Microsc. Microanal. 26, 653-666 (2020)

\section{ACKNOWLEDGEMENTS}

This research has been co-financed by the European Union and Greek national funds through the Operational Program Competitiveness, Entrepreneurship and Innovation, under the call European R \& T Cooperation-Grant Act of Hellenic Institutions that have successfully participated in Joint Calls for Proposals of European Networks ERA NETS (National project code: GRAPH-EYE T8EPA2-00009 and European code: 26632 FLAGERA). L.M., G.Ko. and G.Ki. acknowledge funding by the Hellenic Foundation for Research and Innovation (H.F.R.I.) under the "First Call for H.F.R.I. Research Projects to support Faculty members and Researchers and the procurement of high-cost research equipment grant" (Project No: HFRI-FM17-3034). GKi, S.P. and G.M.M. acknowledge funding from a research co-financed by Greece and the European Union (European Social Fund-ESF) through the Operational Programme "Human Resources Development, Education and Lifelong Learning 2014-2020" in the context of the project "Crystal quality control of two-dimensional materials and their heterostructures via imaging of their non-linear optical properties" (MIS 5050340)". J. $\mathrm{V}$ acknowledges funding from FWO G093417N ('Compressed sensing enabling low dose imaging in transmission electron microscopy') from the Flanders Research Fund EU. J.V. and N.G. acknowledge funding from the European Union under the Horizon 2020 programme within a contract for Integrating Activities for Advanced Communities No 823717-ESTEEM3. J.V. N.G. and A.O. acknowledge funding through a GOA project "Solarpaint" of the University of Antwerp.

\section{AUTHOR CONTRIBUTIONS}

S.P. and G.M. performed the P-SHG experiments and data analysis. L.M. performed the theoretical calculations and data analysis. G.Ko. prepared and characterized the samples. A.O, D.J., and N.G. performed the TEM experiments and data analysis. All authors contributed to the discussion and preparation of the manuscript.

\section{COMPETING INTERESTS}

The authors declare no competing interests.

\section{ADDITIONAL INFORMATION}

Supplementary information The online version contains supplementary material available at https://doi.org/10.1038/s41699-021-00258-5.

Correspondence and requests for materials should be addressed to S. Psilodimitrakopoulos or E. Stratakis.

Reprints and permission information is available at http://www.nature.com/ reprints

Publisher's note Springer Nature remains neutral with regard to jurisdictional claims in published maps and institutional affiliations.

\footnotetext{
Open Access This article is licensed under a Creative Commons Attribution 4.0 International License, which permits use, sharing, daptation, distribution and reproduction in any medium or format, as long as you give appropriate credit to the original author(s) and the source, provide a link to the Creative Commons license, and indicate if changes were made. The images or other third party material in this article are included in the article's Creative Commons license, unless indicated otherwise in a credit line to the material. If material is not included in the article's Creative Commons license and your intended use is not permitted by statutory regulation or exceeds the permitted use, you will need to obtain permission directly from the copyright holder. To view a copy of this license, visit http://creativecommons. org/licenses/by/4.0/.
}

(c) The Author(s) 2021 